\title{
BMJ Open More GP contacts and poorer health of informal caregivers with low socioeconomic status in Germany: results from the population-based DEGS1 and the cross-sectional GPCare-1 study
}

\author{
Boris Gavrilov (D) , Manuela Schmidt, Stefanie Kasten, Samira Sommer, \\ Carmen Hunzelar, Florian Bockheim, Joana Paños-Willuhn, Luisa Offenberg, \\ Maja Oberholz, Nur Ikar, Birgitta Weltermann
}

To cite: Gavrilov B, Schmidt M, Kasten S, et al. More GP contacts and poorer health of informal caregivers with low socioeconomic status in Germany: results from the population-based DEGS1 and the cross-sectional GPCare-1 study. BMJ Open 2021;11:e053146. doi:10.1136/ bmjopen-2021-053146

- Prepublication history for this paper is available online To view these files, please visit the journal online (http://dx.doi. org/10.1136/bmjopen-2021 053146).

Received 05 May 2021 Accepted 22 November 2021

A Check for updates

(C) Author(s) (or their employer(s)) 2021. Re-use permitted under CC BY-NC. No commercial re-use. See rights and permissions. Published by BMJ.

Institute of General Practice and Family Medicine, University Hospital Bonn, Bonn, Germany

Correspondence to

Boris Gavrilov;

hausarztmedizin@ukbonn.de

\section{ABSTRACT}

Objectives Informal caregivers are known to have poorer mental health. Risk factors for caregiver burden include low education, female gender, cohabitation with the care recipient and lack of resources. General practitioners (GPs) have an important role in supporting caregivers. Drawing on data from two surveys, associations between caregivers' socioeconomic status (SES), psychophysical health and GP contacts are analysed.

Design Cross-sectional study. The study draws on data from two surveys (German Health Interview and Examination Survey for Adults, DEGS1 and General Practice Care-1, GPCare-1).

Setting Germany.

Participants DEGS1: German general population (18+ years) $\mathrm{n}=7987$. GPCare-1: general practice patients $(18+$ years $)$ $\mathrm{n}=813$.

Primary outcome Psychophysical health, GP contacts and communication.

Methods Using representative DEGS1 data, the prevalence of informal caregivers, caregivers' burden, chronic stress, various health conditions and frequency of GP contacts were evaluated stratified by SES. Data from the GPCare-1 study addressed caregivers' experiences and communication preferences with GPs.

Results In the DEGS1, the prevalence of caregivers was $6.5 \%$. Compared with non-caregivers, caregivers scored significantly higher for chronic stress (15.45 vs 11.90 ), self-reported poor health (37.6\% vs $23.7 \%$ ) and GP visits last year (3.95 vs 3.11), while lifestyle and chronic diseases were similar. Compared with caregivers with medium/high SES, those with low SES had a significantly lower prevalence of high/medium caregiver burden $(47.9 \%$ vs $67.7 \%$ ) but poorer self-reported health $(56.9 \%$ vs $33.0 \%$ ), while other characteristics did not differ. In the GPCare-1 study, the prevalence of caregivers was $12.6 \%$. The majority of them felt that their GP takes their problems seriously $(63.6 \%)$ without difference by SES.

Conclusion Caregivers with low SES constitute an especially high-risk group for psychological strain, requiring special GP attention to support their needs.
Strengths and limitations of this study

- This study determined the prevalence of informal caregivers using data from a representative population-based survey.

- The cross-sectional nature of both data sets did not allow for the analyses of cause-and-effect relationships.

- Informal caregivers with low socioeconomic status were newly outlined as a risk group for poorer health outcomes

- The COVID-19 pandemic impaired recruitment as unnecessary general practitioners visits were avoided which was compensated by an extension of the data collection period.

\section{INTRODUCTION}

In recent years, family and other informal caregivers (CGs) have been identified as a vulnerable group with poorer subjective health, higher stress and higher prevalence of various risk factors and chronic diseases. ${ }^{1-3}$ Studies have shown that they are at higher risk for adverse mental outcomes including depressive symptoms and depression. ${ }^{2}{ }^{4}$ Moreover, CGs were shown to have deficits in healthy lifestyle, such as smoking, alcohol consumption and physical inactivity. ${ }^{2}$ In some studies, lower self-care behaviour and the risk for social isolation were reported. ${ }^{5}$

In line with studies from other countries, a German study of 2944 participants in need of care and their CGs showed that approximately $83 \%$ of CGs feel rather strongly or very strongly overburdened by their task. ${ }^{6}$ The level of CGs' psychological strain is influenced by various factors. For example, strain is higher when caring for a relative with dementia. $^{7}$ Additional risk factors for high 
CG burden include female gender, low education, cohabitation with the care recipient, high number of hours of care, social isolation, less coping strategies, financial stress and lack of choice in being a caregiver. ${ }^{15}$ 8-10 As multifactorial intervention strategies were shown to provide support, ${ }^{11}{ }^{12}$ informal CGs need to be identified. Yet, the mere identification can be a challenge, as not all CGs describe themselves as caregivers, but define themselves primarily in relation to the person they care for. ${ }^{13}$ Therefore, CGs are also called 'hidden patients'. ${ }^{13}$ Due to their knowledge of family settings, general practitioners (GPs) play an important role in identifying and supporting CGs ${ }^{14}$ yet non-detection might be increased by GPs' hesitancy to address social and economic factors, a lthough these are known to be essential determinants of health status. ${ }^{15}$

In general, the relationship between socioeconomic status (SES) and health is well described in national and international studies. In Germany, the difference in life expectancy between people with low and high SES is 5-10 years. ${ }^{16}$ While correlations between education, income, health status and mortality were studied in various populations, ${ }^{1718}$ few studies address the role of SES or income and health outcomes in CGs. ${ }^{19}{ }^{20}$ In a population-based Brazilian study with 176 elderly, Neri et $^{2 l^{11}}$ showed a strong inverse correlation between family income and depressive symptoms of CGs. ${ }^{21}$ A cross-sectional US study of 246 CGs for dementia patients showed a relation between CGs' SES, subjective CG burden and the number of hours spent caring $^{19}$; the wealthier and higher educated CGs showed significantly higher subjective burden despite fewer hours of caregiving. ${ }^{19}$ A cross-sectional Japanese study of 21584 functionally independent adults aged $\geq 65$ years showed that $8.3 \%$ had caregiving duties: compared with the highest income group, the lowest income group was at least twice as often engaged in caregiving hours $\geq 36$ hours per week. ${ }^{22}$ The risk for depressive symptoms was elevated across income groups in this study, while other studies suggest that CGs with low SES are more likely to suffer from depression. ${ }^{23}$ In addition, reduced access to and use of professional care services was reported from some countries. ${ }^{24} 25$

The study aims to describe the relationship between CGs' SES, psychophysical health and GP contacts. Additionally, data are used to address patients' experiences and communication preferences with their GP on caregiving.

\section{METHODS}

\section{Study design}

The study draws on data from two studies. First, data from the German Health Interview and Examination Survey for Adults (DESG1) were used to describe the prevalence of CGs, degrees of CGs' burden, chronic stress, various health conditions and frequency of GP contacts for the total population and stratified by SES (dataset 1 ). Second, data from the General Practice Care-1 (GPCare-1) study addressed CGs' experiences and communication preferences with their GP (dataset 2). We analysed two data sets to describe informal CGs' GP contacts quantitatively (number of GP visits) and qualitatively (patients' communication experiences with GPs). Using data from the national representative DEGS1, we hypothesised that informal care givers have a poorer psychophysical health and a higher prevalence of GP visits. Additional data from the GPCare-1 exploratively investigated communication experiences of patients with informal caregiving.

\section{Dataset 1: representative DEGS1}

The DEGS1 was carried out by the Robert Koch Institute (RKI) from 2008 to 2011 and is part of the German health monitoring system. It is representative for the German general adult population. ${ }^{26}$ Details about the design and concept are published elsewhere. ${ }^{26-28}$

The survey incorporated self-administered questionnaires, standardised computer-assisted personal interviews and examinations, laboratory analyses as well as tests of physical and cognitive function. ${ }^{28-31}$ The target population was German residents between 18 and 79 years and consisted of 7987 adult participants. Datasets of the DEGS1 are available for public use on request. To allow for representative conclusions regarding the German adult population, data are adjusted using survey-specific weighting factors. ${ }^{27} 2831$

DEGS1 variables: sociodemographic, health and CG characteristics Sociodemographic parameters: the following items were used for analysis:

- Age, gender, marital status (married, single, divorced, widowed), number of persons in household.

- To calculate the multidimensional SES index (SES index) in three categories (low/middle/high), information on the highest level of education, job and monthly income were used. This categorisation is based on the international classification 'Comparative Analyses of Social Mobility in Industrial Nations. ${ }^{32} 33$

Health, health behaviour and health utilisation parameters: The following items were used for the analysis:

- Participants' subjective health status had been obtained using a 5-point Likert Scale (very good health to very poor health); for this analysis, data were dichotomised (very good/good health vs middle/ poor/very poor health).

- Self-reported chronic disease (at least one), history of physician-diagnosed depression, current therapy for depression, current depressive symptoms (Patient Health Questionnaire-2 (PHQ-2]; categorised) ${ }^{34}$ and obesity.

- The following health behaviours were requested: smoking, risky consumption of alcohol; sport activity as number of hours per week (no sport, $<2$ hours, 2-4hours, more than 4 hours).

- Chronic stress had been measured with the Strategies for Coping with Stress Scale (SCSS; score from 1 to 48 ), which was categorised as follows: 1-11=low stress, $12-22=$ middle stress, $>22=$ high stress. The 
subgroups were calculated following the original DEGS1 approach. ${ }^{35}$

- Number of GP visits during the past 12 months.

Parameters addressing CGs and CGs' burden: The following items were used:

- Participants' information if they are informal CGs.

- If yes: participants were asked about the associated burden categorised in five levels (no burden, little burden, medium burden, high burden and very high burden); for this analysis, this was dichotomised into none/little/medium burden and strong/very strong burden.

Dataset 2: GPCare-1 study in GP practices addressing patients' experiences and communication preferences with their GP Practice and patient recruitment and involvement

Practices of the teaching practice network of the Institute of General Practice and Family Medicine of the University of Bonn, Bonn, Germany, were asked for participation between June and August 2020. Based on population data for practice locations from the statistical state offices of North Rhine-Westphalia and Rhineland-Palatinate, selected sampling was used to ensure that participants of different age, income categories, education levels and migration background were included.

The two-sided questionnaire was made available in four languages (German, English, Turkish, Arabic) and included a study information addressing the study aim, voluntary participation, data management and the anonymity of the survey. The questionnaire was pretested with 40 volunteers from the general population with subsequent minor adjustments. Practice staff and-if wished by the practice-study personnel informed patients about the study. Patients were asked to fill the questionnaire in the waiting room prior to their GP appointment. Once filled, they sealed the questionnaire in an envelope and placed it in a study letter box in the practice. Those not able or willing to fill the questionnaire in the practice, could use the same envelope for a cost-free mail to the study centre at the institute. Patients were eligible to participate when they were at least 18 years old, physically and mentally able to fill the questionnaire, and visited the practice during the time of recruitment.

\section{GPCare-1 study: questionnaire development}

In line with the DEGS1, items for sociodemographic, health and CG characteristics were used similarly in the questionnaire. Additional questions addressed patients' experiences with their GP and communication preferences. To shorten the questionnaire, some items from the DEGS1 were simplified according to categories used in the analysis of the DEGS1 data.

In detail:

- Age and relationship status were requested identical to the DEGS1.

- The third gender was added as answer option.

- Education was requested in three categories (no school education/ secondary school up to 9th/up to 10th grade, high school (A-levels)/vocational school and university degree).

- Income was requested identical to the DEGS1 asking participants to indicate their household net income.

- As in the DEGS1, the PHQ-2 was used to measure depressive symptoms.

- The health status during the past 4 weeks was assessed using the first question from the Short Form 8-Item Health Survey.

The following items were requested in addition:

Number of years with their current GP $(<1$ year, 1-2 years, $3-5$ years and more than 5 years).

Details addressing the physician-patient communication were obtained using eight self-developed statements (5-point Likert scale: strongly agree to strongly disagree), which concerned the current situation and wishes of patients regarding their communication with the GP.

Prior to the study, the questionnaire was piloted in 40 persons from the general population and was found suitable with minor adjustments.

\section{Statistical analysis}

Statistical analyses were performed using the software SPSS V.26. All analyses of the DEGS1 data were weighted using the survey-specific weighting factor based on age, gender, region of residence, level of education, community class and nationality provided by the RKI, to allow for estimates representative of the German population.

Frequency distributions and descriptive estimates were inspected for the entire population. Comparison of the subpopulations of CGs and non-CGs, as well as caregivers stratified by low vs middle/high SES were conducted through $\chi^{2}$ tests for categorical as well as a comparison of the means through t-tests for numerical data. The same approach was used for comparison of the employment situation of CGs with high burden, stratified by SES. All dataset fulfilled the necessary assumptions of these tests.

Multiple linear regression analysis was conducted to estimate how caregiving status and SES are associated with the number of GP contacts, the covariates age, sex and depressive symptoms were included. In order to fulfil all statistical requirements, 92 outliers $(1.1 \%)$ were excluded from the regression analysis due to extreme values regarding GP contacts $(>15)$.

The data from the GPCare-1 study was analysed using the same approach: following a first description and distribution analysis of the sample, group comparisons were conducted for the subpopulations consisting of CGs and non-CGs. Additionally, $\chi^{2}$ analysis was used to compare patients' answers regarding their communication preferences stratified by subgroups.

\section{Practice and patients involvement}

The GPCare-1 questionnaire was pretested in the four languages with 40 native speakers from the general population. Suggestions were integrated in the final version of the questionnaire. Primary care physicians from the institute were involved in the design and conduct of the study. 
Some of the participation researcher had experiences as patients themselves which influence the development of the questionnaire. The teaching practices and patients recruited were not involved in the planning, conduct, reporting or dissemination of this study. Results will be disseminated in scientific context and through patient associations.

\section{RESULTS}

\section{Characteristics of the DEGS population}

The population of the DEGS1 consisted of 7987 participants. Half of the population were females $(50.3 \%)$. The mean age of the participants was 47.4 years (95\% C: 47.02 to 47.83$)$. The majority of the participants had a middle SES $(60.3 \%)$. The mean stress level of the total population was 12.11 (95\% CI 11.85 to 12.38). The mean number of GPs visits in the last 12 months was 3.19 (95\% CI 3.02 to 3.36). The majority of the participants reported their general state of health to be very good/good $(74.7 \%)$. A total of 546 participants were informal CGs $(6.5 \%)$ (table 1).

\section{Comparison of informal CGs and non-CGs in the DEGS1 population}

Informal CGs were more frequently female than male (63.8\% vs $36.2 \%, \mathrm{p}<0.01)$. No significant difference was found for the SES distribution between CGs and noncaregiver, yet non-caregivers indicated a significantly better health status than caregivers (76.3 vs $62.4 \%$, $\mathrm{p}<0.001)$. CGs showed a higher prevalence of having at least one chronic disease $(37.1 \%$ vs $29.7 \%, \mathrm{p}=0.005)$, being diagnosed with depression $(15.9 \%$ vs $11.1 \% \mathrm{p}<0.01)$ and suffering from obesity $(27.8 \%$ vs $22.8 \%, \mathrm{p}=0.040)$. Also, CGs had a significantly higher mean number of GPs' visits than non-CGs (3.95 vs 3.11, $\mathrm{p}<0.001)$. For details see table 2.

The linear regression analysis showed an association between the number of GP visits, SES and depressive symptoms that remained significant after adjustment for gender and age $\left(R^{2}=0.058\right)$. Men, older participants and those with currently more depressive symptoms visited their GP more often. However, there was no significant relationship between being a CG and GPs visits (table 3).

When stratified by SES, CGs with low SES reported significantly less CGs' burden than those with middle/ high SES (52.1\% vs 32.3\%, p=0.02) (figure 1). However, compared with CGs with medium/high SES, those with low SES had a higher prevalence of subjective poor health $(56.9 \%$ vs $33 \%, \mathrm{p}<0.002)$, were more often in therapy for depression $(63,9 \%$ vs $25,9 \%, \mathrm{p}<0.05)$ (figure 1) and showed a higher chronic stress level (16.58 vs 15.21) (table 4). CGs with low SES visited their GP significantly more frequently in the last 12 months (6.08 vs 3.45 , $\mathrm{p}<0.001$ ) (figure 2). Considering the group of CGs with high CG burden, there were no significant differences in employment status and working hours per week between SES groups.
Table 1 DEGS1 participants: sociodemographic and medical characteristics, weighted

\begin{tabular}{|c|c|c|}
\hline & $\begin{array}{l}N^{*} \\
(n=7987)\end{array}$ & $\%^{*}$ \\
\hline Sex (female) & 4198 & 50.3 \\
\hline Age, mean, $\mathrm{Cl}$ & 47.43 & $47.02-47.83$ \\
\hline \multicolumn{3}{|l|}{ Living situation } \\
\hline Married & 5160 & 62.5 \\
\hline Single & 1695 & 26.2 \\
\hline Divorced/widowed & 996 & 11.4 \\
\hline Persons in household, mean, $\mathrm{Cl}$ & 2.69 & $2.63-2.74$ \\
\hline Living alone & 1243 & 15.8 \\
\hline 2 Persons & 3495 & 39.5 \\
\hline 3 Persons & 1424 & 19.2 \\
\hline 4 Persons & 1198 & 16.8 \\
\hline$\geq 5$ Persons & 529 & 8.6 \\
\hline \multicolumn{3}{|l|}{ Socioeconomic status } \\
\hline Low & 1238 & 19.7 \\
\hline Middle & 4743 & 60.3 \\
\hline High & 1916 & 20.0 \\
\hline \multicolumn{3}{|l|}{ Caregiving } \\
\hline Caregivers & 546 & 6.5 \\
\hline \multicolumn{3}{|l|}{ Burden of caregiving: $(n=529)$} \\
\hline Very high/high burden & 138 & 24.3 \\
\hline Medium burden & 203 & 39.8 \\
\hline Little/no burden & 188 & 36.0 \\
\hline \multicolumn{3}{|l|}{ Physical and mental health } \\
\hline Subjective health very good or good & 5815 & 74.7 \\
\hline $\begin{array}{l}\text { Subjective health moderate, poor or very } \\
\text { poor }\end{array}$ & 2082 & 25.3 \\
\hline Has at least one chronic disease & 2575 & 30.5 \\
\hline History of depression & 924 & 11.6 \\
\hline Current symptoms of depression & 536 & 7.7 \\
\hline Chronic stress, mean, Cl & 12.11 & $11.85-12.38$ \\
\hline \multicolumn{3}{|l|}{ Lifestyle } \\
\hline Smoker & 2115 & 29.8 \\
\hline Risky consumption of alcohol & 2517 & 33.6 \\
\hline Obesity & 1702 & 23.5 \\
\hline \multicolumn{3}{|l|}{ Sport activity (hours per week) } \\
\hline No sport & 2524 & 33.7 \\
\hline$<2$ hours & 3286 & 40.9 \\
\hline $2-4$ hours & 1203 & 15.3 \\
\hline$>4$ hours & 727 & 10.1 \\
\hline \multicolumn{3}{|l|}{ GP contacts } \\
\hline Visited a GP in last 12 months & 6231 & 79.4 \\
\hline No of GP visits in last 12 months, mean, $\mathrm{Cl}$ & 3.19 & $3.02-3.36$ \\
\hline
\end{tabular}

\section{Characteristics of the GPCare-1 population}

The total population consisted of 813 patients from 12 general practices. The majority of the participants were 
Table 2 DEGS1 participants: comparison of sociodemographic and medical characteristics between caregivers and noncaregivers, weighted

\begin{tabular}{|c|c|c|c|c|c|}
\hline & \multicolumn{2}{|c|}{ Caregivers } & \multicolumn{2}{|c|}{ Non-caregivers } & \multirow[b]{2}{*}{$P$ value } \\
\hline & $\begin{array}{l}N^{*} \\
(n=546)\end{array}$ & $\%^{*}$ & $\begin{array}{l}N^{*} \\
(n=7135)\end{array}$ & $\%^{*}$ & \\
\hline Sex (female) & 375 & 63.8 & 3673 & 49.5 & $<0.001$ \\
\hline Age, mean, $\mathrm{Cl}$ & 52.90 & $51.19-54.61$ & 46.67 & $46.25-47.10$ & $<0.001$ \\
\hline Living situation & & & & & $<0.001$ \\
\hline Married & 403 & 70.2 & 4621 & 61.9 & \\
\hline Single & 59 & 14.1 & 1606 & 27.4 & \\
\hline Divorced/widow & 83 & 15.7 & 863 & 10.8 & \\
\hline Number of persons in household, mean, $\mathrm{Cl}$ & 2.65 & $2.51-2.78$ & 2.69 & $2.63-2.75$ & 0.504 \\
\hline Living alone & 70 & 12.7 & 1111 & 15.8 & \\
\hline 2 Persons & 255 & 43.2 & 3132 & 39.2 & \\
\hline 3 Persons & 105 & 21.3 & 1292 & 19.2 & \\
\hline 4 Persons & 79 & 14.7 & 1109 & 17.3 & \\
\hline$\geq 5$ Persons & 37 & 8.1 & 453 & 8.4 & \\
\hline Socioeconomic status & & & & & 0.138 \\
\hline Low & 89 & 19.4 & 1061 & 18.6 & \\
\hline Middle & 353 & 64.3 & 4280 & 60.6 & \\
\hline High & 103 & 16.3 & 1786 & 20.8 & \\
\hline \multicolumn{6}{|l|}{ Burden of caregiving } \\
\hline Very high/high burden & 138 & 24,3 & & & \\
\hline Medium burden & 203 & 39.8 & & & \\
\hline Little/no burden & 188 & 36.0 & & & \\
\hline \multicolumn{6}{|l|}{ Physical and mental health } \\
\hline Subjective health very good or good & 342 & 62.4 & 5342 & 76.3 & $<0.001$ \\
\hline Subjective health moderate, poor or very poor & 201 & 37.6 & 1763 & 23.7 & \\
\hline Has at least one chronic disease & 218 & 37.1 & 2248 & 29.7 & 0.005 \\
\hline History of depression & 89 & 15.9 & 793 & 11.1 & 0.010 \\
\hline Current symptoms of depression & 45 & 6.1 & 461 & 7.4 & 0.488 \\
\hline Stress scale, mean, $\mathrm{Cl}$ & 15.45 & $14.40-16.50$ & 11.90 & $11.63-12.16$ & $<0.001$ \\
\hline \multicolumn{6}{|l|}{ Lifestyle } \\
\hline Smoker & 126 & 25.0 & 1925 & 30.1 & 0.072 \\
\hline Risky consumption of alcohol & 143 & 27.6 & 2348 & 34.0 & 0.021 \\
\hline Obesity & 136 & 27.8 & 1494 & 22.8 & 0.040 \\
\hline Sport activity & & & & & 0.052 \\
\hline No sport & 193 & 38.3 & 2297 & 33.3 & \\
\hline$<2$ hours & 240 & 41.9 & 3000 & 40.9 & \\
\hline $2-4$ hours & 73 & 12.3 & 1118 & 15.5 & \\
\hline$>4$ hours & 36 & 7.4 & 682 & 10.3 & \\
\hline \multicolumn{6}{|l|}{ GP contacts } \\
\hline Visited a GP in the last 12 months & 457 & 83.4 & 5682 & 93.3 & 0.090 \\
\hline No of GP visits in the last 12 months, mean, $\mathrm{Cl}$ & 3.95 & $3.20-4.69$ & 3.11 & $2.95-3.27$ & $<0.001$ \\
\hline
\end{tabular}

${ }^{*} \mathrm{n}$ or percent unless noted otherwise.

DEGS1, German Health Interview and Examination Survey for Adults; GP, general practitioner. 
Table 3 DEGS1 participants: multiple regression analysis for number of GP visits during the last year

\begin{tabular}{|c|c|c|c|c|}
\hline Parameter & Estimate & SE & $95 \% \mathrm{Cl}$ & $P$ value \\
\hline Constant & 1.191 & 0.150 & 0.896 to 1.486 & $<0.001$ \\
\hline Low SES (Ref. Middle/high SES) & 0.535 & 0.122 & 0.294 to 0.0776 & $<0.001$ \\
\hline Female (Ref. Male) & -0.314 & 0.079 & -0.470 to -0.158 & $<0.001$ \\
\hline Depressive symptoms & 0.403 & 0.044 & 0.317 to 0.489 & $<0.001$ \\
\hline $\mathrm{R}^{2} \mathrm{Adj}$ & $5.8 \%$ & & & \\
\hline
\end{tabular}

Dependent variable: number of GP visits during the last year.

DEGS1, German Health Interview and Examination Survey for Adults; GP, general practitioner; SES, socioeconomic status.

female (59.3\%) and the mean age was 51.6 years (SD 18.7). As $24.2 \%$ of the patients did not report their income, the education level was used to classify SES. The majority of participants had a middle education level (low 32.0\%, middle $43.5 \%$, high $24.6 \%$ ). In the patient population, $12.6 \%(\mathrm{n}=98)$ were current CGs. For details see table 5.

\section{Differences between CGs and non-CGs from GP practices}

CGs differed from non-CGs significantly regarding several characteristics: caregivers were more likely to be female (69.1 vs 57.7\%) and in a self-reported medium/poor/very poor state of health $(55.7 \%$ vs $39.6 \%, \mathrm{p}=0.003)$. Almost twice as many CGs had a history of depression (caregivers: 25.5\%; non-CGs: $13.4 \%$, $\mathrm{p}=0.002$ ), while current depressive symptoms did not differ between groups (23.9\% vs $25.6 \%, \mathrm{p}=0.73$ ). Additionally, the prevalence of high chronic stress measured by the SCSS was almost twice as high among CGs $(48.1 \%$ vs $27.3 \%, \mathrm{p}<0.001)$. There was no significant difference in educational level between CGs and non-CGs $(\mathrm{p}=0.531)$.

Regarding communication with their GP, about $65 \%$ of CGs and non-CGs report that their GP makes them feel comfortable talking about sensitive issues $(64.4 \%$ vs $66.5 \%)$ and asks about their personal strains $(53.4 \%$ vs $52.9 \%$ ). Also, in both groups more than $60 \%$ felt that their GPs takes their problems very seriously $(63.6 \%$ vs $72.7 \%, \mathrm{p}=0.211)$. While the majority in both groups
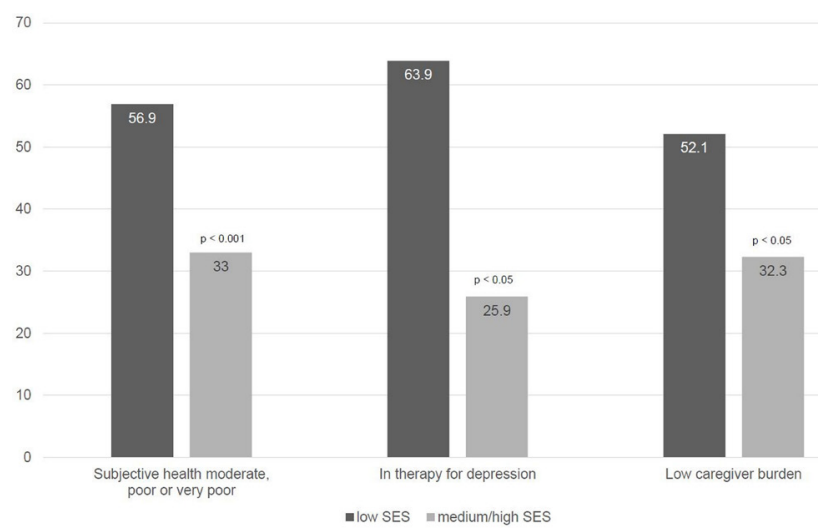

Figure 1 DEGS1: Health status of informal caregivers by SES. SES, socioeconomic status. prefers to be rather asked directly about their personal strains $(46.6 \%$ vs $40.8 \% \mathrm{p}=0.355)$, more CGs indicated that they would rather prefer a questionnaire about their personal strains (39.8\% vs non-CGs: $26.5 \%, \mathrm{p}=0.035)$. For details see table 5 .

\section{DISCUSSION}

In line with the international literature, our analysis of the population-based, representative German DEGS1 data showed that CGs are a population at high risk for adverse mental outcomes: Compared with non-CGs, CGs showed significantly higher prevalence of poor subjective health, high chronic stress, depression and more GP visits during the last year. Focusing on SES, our study is one of the few that identified caregivers with low SES as a very high-risk group when compared with those with medium/high SES: those with low SES reported even poorer subjective health, were more likely to receive therapy for depression and had more GP visits during the last year. Yet, the prevalence of high CG burden was significantly lower in this very high-risk group when compared to those with medium/high SES.

The finding of an inverse relationship of CG burden and SES was also reported in studies from other countries with differing healthcare and social systems. For example, CGs' education (which was used as a surrogate for SES) was associated with higher CG burden but fewer CG hours. ${ }^{19}$ One assumption is that individuals with higher SES have less time for caregiving due to their professional obligations. Other possible explanations are differences in coping strategies between SES groups ${ }^{19}$ as well as different subjective perceptions of the caregiving burden. ${ }^{36}$ A 2020 German online-survey among 1000 CGs showed that about $50 \%$ of respondents wished more support. ${ }^{37}$ Stratified by SES, working CGs and those with a high education level wished slightly, but significantly more support. In contrast, those living in the same household with the care recipient and those with high household income scored slightly underproportionally. ${ }^{37}$ In contrast, our analysis which is based on population-based data showed no association between CG burden and employment when stratified by SES. 
Table 4 DEGS1 caregivers: comparison of sociodemographic and medical characteristics between low and middle/high SES, weighted

\begin{tabular}{|c|c|c|c|c|c|}
\hline \multirow[b]{2}{*}{ Variable } & \multicolumn{2}{|c|}{ Low SES } & \multicolumn{2}{|c|}{ Middle/high SES } & \multirow[b]{2}{*}{$P$ value } \\
\hline & $\mathbf{N}^{*}$ & $\%^{*}$ & $\mathbf{N}^{*}$ & $\%$ & \\
\hline Sex (female) & 6 & 69.3 & 310 & 62.4 & 0.393 \\
\hline Age, mean, $\mathrm{Cl}$ & 54.38 & $50.73-58.03$ & 52.54 & $50.64-54.44$ & 0.255 \\
\hline Living situation & & & & & 0.042 \\
\hline Married & 58 & 65.8 & 343 & 71.2 & \\
\hline Single & 8 & 8.8 & 51 & 15.4 & \\
\hline Divorced/widow & 23 & 25.4 & 60 & 13.4 & \\
\hline No of persons in household, mean, $\mathrm{Cl}$ & 2.79 & $2.50-3.07$ & 2.61 & $2.47-2.75$ & 0.210 \\
\hline Living Alone & 12 & 13.5 & 58 & 12.5 & \\
\hline 2 Persons & 38 & 35.1 & 216 & 45.1 & \\
\hline 3 Persons & 20 & 22.0 & 85 & 21.1 & \\
\hline 4 Persons & 12 & 17.9 & 67 & 14.0 & \\
\hline$\geq 5$ Persons & 7 & 11.5 & 30 & 7.4 & \\
\hline Burden of caregiving & & & & & 0.022 \\
\hline Very high/high burden & 16 & 22.4 & 122 & 24.8 & \\
\hline Medium burden & 23 & 25.5 & 179 & 42.9 & \\
\hline Little/No burden & 47 & 52.1 & 141 & 32.3 & \\
\hline \multicolumn{6}{|l|}{ Physical and mental health } \\
\hline Subjective health very good or good & 39 & 43.1 & 302 & 67.0 & 0.002 \\
\hline Subjective health moderate, poor or very poor & 50 & 56.9 & 151 & 33.0 & \\
\hline Has at least one chronic disease & 44 & 40.8 & 174 & 36.1 & 0.513 \\
\hline History of depression & 14 & 14.6 & 75 & 16.2 & 0.711 \\
\hline Current symptoms of depression & 8 & 10.3 & 37 & 7.8 & 0.513 \\
\hline Stress scale, mean, $\mathrm{Cl}$ & 16.58 & $13.83-19.32$ & 15.21 & $14.09-16.32$ & 0.276 \\
\hline \multicolumn{6}{|l|}{ Lifestyle } \\
\hline Smokers & 25 & 32.5 & 101 & 23.2 & 0.100 \\
\hline Risky consumption of alcohol & 20 & 19.6 & 123 & 29.5 & 0.143 \\
\hline Obesity & 21 & 22.9 & 115 & 29.0 & 0.379 \\
\hline Sport activity (hours per week) & & & & & 0.157 \\
\hline No sport & 39 & 50.1 & 154 & 35.5 & \\
\hline$<2$ hours & 33 & 32.9 & 206 & 44.0 & \\
\hline $2-4$ hours & 12 & 12.1 & 61 & 12.4 & \\
\hline$>4$ hours & 5 & 4.9 & 31 & 8.1 & \\
\hline \multicolumn{6}{|l|}{ GP contacts } \\
\hline Visited a GP in the last 12 months & 80 & 88.9 & 376 & 82.1 & 0.283 \\
\hline No of visits in the last 12 months, mean, $\mathrm{Cl}$ & 6.08 & $3.16-9.01$ & 3.45 & $2.99-3.90$ & $<0.001$ \\
\hline
\end{tabular}

${ }^{*} \mathrm{n}$ or per cent unless noted otherwise.

DEGS1, German Health Interview and Examination Survey for Adults; GP, general practitioner; SES, socioeconomic status.

In line with prior studies, our analysis of the DEGS1 data showed a significant higher prevalence of depression among CGs than non-CGs, yet there was no significant difference when stratified by low vs medium/high SES. This is in contrast to findings from a Brazilian study by Neri $e t a l^{21}$ which showed a higher prevalence of depression among the poorest participants. Differences in the countries' social systems likely play a role: various health, social and financial support structures for care recipients and care givers are available in Germany, for example, nursing insurance is part of the statuary health insurance. In addition, methodological differences may play a role as Neri $e$ a $a .^{21}$ only used income, while the DEGS1 combined various information to calculate SES. ${ }^{21}$ 


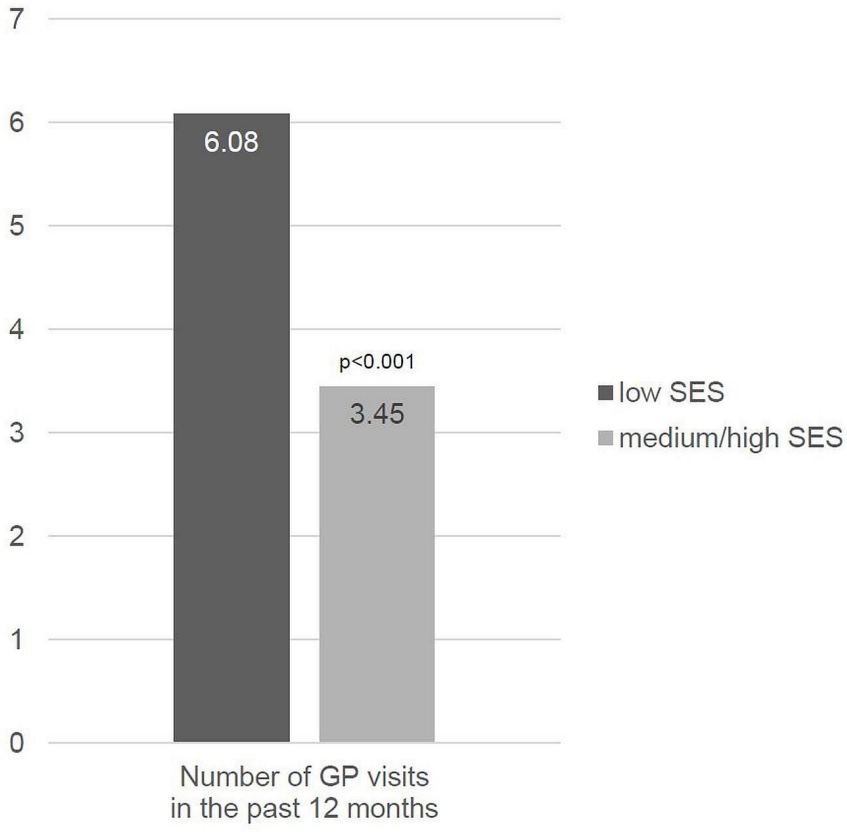

Figure 2 DEGS1: Number of GP visits of caregivers with low versus medium/high SES. GP, general practitioner; SES, socioeconomic status.

In addition to the higher prevalence of depression, CGs in the DEGS1 showed significantly higher rates of chronic stress and having at least one chronic disease compared with non-CGs. These findings are in line with other studies, yet it is important to note that a long held narrative that this psychological strain is linked to poorer health and increased mortality is not supported by several prospective studies. ${ }^{36}{ }^{38}{ }^{39}$ Based on more than 3500 US family CGs, the REGARDS (Reason for Geographic and Racial Differences in Stroke) study showed that CGs had a $16.5 \%$ lower mortality than non-CGs (HR 0.83 ) after a 7-year follow-up. ${ }^{40}$ Depressive symptoms and perceived high strain were associated with higher mortality in nonCGs, but not in CGs. ${ }^{40}$ These findings led to the 'stressbuffering hypothesis' of caregiving ${ }^{40}$ which suggests that caregiving is similar to other prosocial helping activities which ameliorate the adverse impact of other factors on mortality.

While findings on psychological well-being are consistent between studies, contradictory results are reported with regard to health behaviour of CGs. In the DEGS1, caregivers were significantly less likely than non-CGs to engage in risky drinking. Beesley et $a t^{41}$ studied CGs of 101 women with ovarian cancer and showed that more than half of the CGs experienced negative lifestyle and weight changes after becoming a caregiver. ${ }^{41}$ Similarly, our findings indicate that CGs had a higher prevalence of obesity which could be explained by the inability to maintain healthy habits as a consequence of the emotional and physical demands imposed by their caregiving duties.

According to the DEGS1 data, over $80 \%$ of CGss and non-CGs visited a GP during the last year. While bivariate analyses of the DEGS1 data showed that caregivers had significantly more GP visits during the last year than non-caregivers, no significant relationship was found in the regression model after adjusting for gender, age and depressive symptoms. Interestingly, caregivers with low SES had a significantly higher prevalence of current therapy for depression which indicates that GPs offer support. This is in line with results of our GPCare-1 study: more than $50 \%$ of patients reported that their GP asks for their personal strains, and more than $60 \%$ reported that their GP makes them feel comfortable in discussing sensitive issues and then they felt to be taken seriously. This is of utmost importance as GPs have the opportunity to address the burden of caregiving and help to prevent adverse mental outcomes by providing emotional support and access to supportive services, for example, various CG support approaches offered by the German statutory nursing insurances. Although the majority of patients wished to talk to their GP directly, more CGs than nonCGs indicated to accept a questionnaire to address their strain. This new finding warrants further studies to be understood more in detail.

\section{Strengths and limitations}

First, it is noteworthy that the population-based nature of the DEGS1 is a strength as it allows for the description of CGs on a population base, that is, irrespective of specific caregiving disease contexts and potential selection biases as in many other studies. Second, the additional GPCare-1 study complements these data with information on patients' communication preferences. Limitations are the lack of details on care situations such as the amount of care needed, the emotional relationship between CGs and recipients, and the conditions requiring care. Furthermore, the cross-sectional nature of both surveys does not allow for the analysis of cause-and-effect relationships regarding depression and caregiving which requires prospective studies. And last, within the GPCare-1 study, education rather than SES as in the DEGS1 was used because of about $20 \%$ missing data on household income. Unexpectedly, the COVID-19 pandemic impaired recruitment as unnecessary GP visits were avoided by patients, which was compensated by an extension of the data collection period.

\section{CONCLUSION AND PERSPECTIVES}

It is important for GPs to be aware that CGs' are at increased risk for adverse mental outcomes. Our study identified CGs with low SES as a very high-risk group when compared with those with medium/high SES which was irrespective of employment. When asked for communication preferences, more CGs wished to be personally addressed by their GP rather than fill a questionnaire on their condition yet filling a questionnaire was accepted by more than one-third of patients with caregiving responsibilities. It is important for GPs to be aware that CGs' are at increased risk for adverse mental health outcomes. Our study identified CGs with low SES 
Table 5 GPCare-1 study: characteristics of total population and comparison between caregivers and non-caregivers

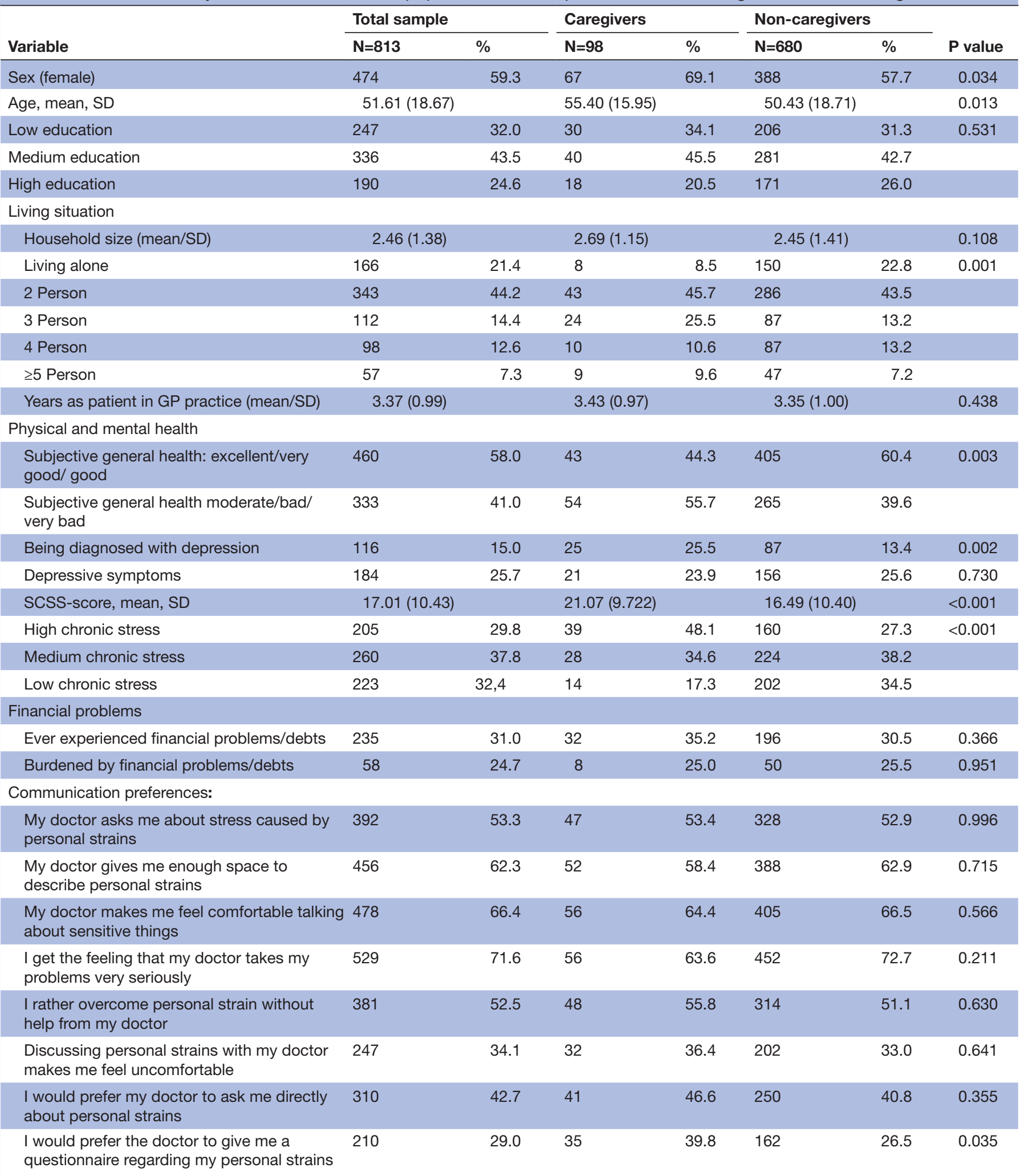

n or per cent unless noted otherwise.

GP, general practitioner; GPCare-1, General Practice Care-1 study; SCSS, Strategies for Coping with Stress Scale.

as a very high-risk group when compared with those with medium/high SES which was irrespective of employment. Given the high psychosocial burden associated with informal caregiving, a respective screening in GP practices should be implemented. In ageing societies such as Germany, additional formal and informal 
support networks may be required to better support informal CGs.

Acknowledgements We owe special thanks to the Robert Koch Institute, Berlin, for kindly providing the data set and additional information on the DEGS1 survey. We are especially grateful to Dr. Franziska Prütz, Robert Koch Institute, for her critical review of the manuscript. Also, we thank the participating practices of the research practice network of the Institute of General Practice and Family Medicine, University of Bonn, and their patients for their friendly participation. Furthermore, we thank the volunteers from the general populations for pre-testing the questionnaire. The study was performed within the framework of the dissertation program of the Institute of General Practice and Family Medicine which was kindly supported by the Medical Faculty of the University of Bonn.

Contributors BG, BW, SS and SK developed the study question and study design. $B G$ and MS developed the statistical approach and analysed the data. BG, BW, MS, SS and SK interpreted the data and results. BG, BW, SS, SK, CH, FB, MO, LO, NI and JP-W conceptualised the GPCare-1 questionnaire, recruited GPs, collected data and added substantial inputs by critically reviewing and revising the draft manuscripts for improvement. BW is responsible for the overall content as guarantor. All authors read and approved the final manuscript.

Funding The GPCare-1 study was funded by the Institute of General Practice and Family Medicine.

Competing interests None declared.

Patient and public involvement Patients and/or the public were involved in the design, or conduct, or reporting, or dissemination plans of this research. Refer to the Methods section for further details.

Patient consent for publication Not applicable.

Ethics approval The DEGS1 survey had been approved by the Charite Universitätsmedizin Berlin Ethics' Committee (No. EA2/047/08). Participants provided written informed consent before the interview and examination. The Ethics Committee of the Medical Faculty of the University of Bonn provided ethical approval for the GPCare-1 primary data collection in June, 2020 (№. 215/20). The GP patient study was registered in the German Clinical Trials Register (DRKS00022330). Patients received information on study procedures, confidentiality, anonymity, and were informed that participation was voluntary both verbally and in writing. Thus, no formal written consent was required as the return of the anonymous questionnaire indicated informed consent from the patient for their data to be used in the study.

Provenance and peer review Not commissioned; externally peer reviewed.

Data availability statement Data are available on reasonable request. The DEGS1 data set is available on application to scientists as an anonymised dataset for secondary data analyses at the Robert Koch Institute (RKI). For further information contact datennutzung@rki.de. The GPCare-1 data are available on reasonable request to the Institute of General Practice and Family Medicine of the University of Bonn, Germany.

Open access This is an open access article distributed in accordance with the Creative Commons Attribution Non Commercial (CC BY-NC 4.0) license, which permits others to distribute, remix, adapt, build upon this work non-commercially, and license their derivative works on different terms, provided the original work is properly cited, appropriate credit is given, any changes made indicated, and the use is non-commercial. See: http://creativecommons.org/licenses/by-nc/4.0/.

\section{ORCID iD}

Boris Gavrilov http://orcid.org/0000-0003-1699-193X

\section{REFERENCES}

1 Adelman RD, Tmanova LL, Delgado D, et al. Caregiver burden: a clinical review. JAMA 2014;311:1052-9.

2 von Känel R, Mausbach BT, Dimsdale JE, et al. Effect of chronic dementia caregiving and major transitions in the caregiving situation on kidney function: a longitudinal study. Psychosom Med 2012;74:214-20.

3 Zwerling JL, Cohen JA, Verghese J. Dementia and caregiver stress. Neurodegener Dis Manag 2016;6:69-72.

4 Gallagher D, Rose J, Rivera P, et al. Prevalence of depression in family caregivers. Gerontologist 1989;29:449-56.
5 Rodakowski J, Skidmore ER, Rogers JC, et al. Role of social support in predicting caregiver burden. Arch Phys Med Rehabil 2012;93:2229-36.

6 Schneekloth U. Tendency of development and perspectives of domestic care. Core results of the potential and limits of independent living (MuG III) study. Z Gerontol Geriatr 2006;39:405-12.

7 Gräßel E. Häusliche Pflege dementiell und nicht dementiell ErkrankterTeil II: Gesundheit und Belastung der Pflegenden. Zeitschrift für Gerontologie und Geriatrie 1998;31:57-62.

8 Gallicchio L, Siddiqi N, Langenberg P, et al. Gender differences in burden and depression among informal caregivers of demented elders in the community. Int J Geriatr Psychiatry 2002;17:154-63.

9 Salmon JR, Kwak J, Acquaviva KD, et al. Transformative aspects of caregiving at life's end. J Pain Symptom Manage 2005;29:121-9.

10 Schulz R, Beach SR, Cook TB, et al. Predictors and consequences of perceived lack of choice in becoming an informal caregiver. Aging Ment Health 2012;16:712-21.

11 Chiu MYL, Wei GFW, Lee S, et al. Empowering caregivers: impact analysis of FamilyLink education programme (FLEP) in Hong Kong, Taipei and Bangkok. Int J Soc Psychiatry 2013;59:28-39.

12 Ducharme F, LéVesque L, Gendron L, et al. Development process and qualitative evaluation of a program to promote the mental health of family caregivers. Clin Nurs Res 2001;10:182-201.

13 Höppner C, Schneemilch M, Lichte T. Pflegende Angehörige und ihre Belastungen in Hausarztpraxen identifizieren - Hindernisse und Empfehlungen. Zeitschrift für Allgemeinmedizin 2015;91:310-4.

14 Grunfeld E, Coyle D, Whelan T, et al. Family caregiver burden: results of a longitudinal study of breast cancer patients and their principal caregivers. CMAJ 2004;170:1795-801.

15 Moscrop A, MacPherson P. Should doctors record their patients' income? British Journal of General Practice 2014;64:e672-4.

16 Lampert T, Saß AC, Häfelinger M. soziale Ungleichheit und Gesundheit. Berlin: Gesundheitliche Auswirkungen von Einkommensungleichheit, 2005.

17 Lampert T, Kroll L, Müters S, et al. Messung des sozioökonomischen status in Der studie Zur Gesundheit Erwachsener in Deutschland (DEGS1). Bundesgesundheitsblatt Gesundheitsforschung Gesundheitsschutz 2013;56:631-6.

18 Mielck A. Soziale Ungleichheit und Gesundheit. Empirische Belege für die zentrale Rolle der schulischen und beruflichen Bildung. In: Brähler E, Kiess J, Schubert C, eds. Gesund und gebildet: Voraussetzungen für eine moderne Gesellschaft. Göttingen: Vandenhoeck \& Ruprecht, 2012: 129-45.

19 Hughes TB, Black BS, Albert M, et al. Correlates of objective and subjective measures of caregiver burden among dementia caregivers: influence of unmet patient and caregiver dementiarelated care needs. Int Psychogeriatr 2014;26:1875-83.

20 Posch-Eliskases U, Rungg C, Moosbrugger M, et al. Stress bei pflegenden Angehörigen. HeilberufeScience 2015;6:27-32.

21 Neri AL, Yassuda MS, Fortes-Burgos ACG, et al. Relationships between gender, age, family conditions, physical and mental health, and social isolation of elderly caregivers. Int Psychogeriatr 2012;24:472-83.

22 Saito T, Kondo N, Shiba K, et al. Income-based inequalities in caregiving time and depressive symptoms among older family caregivers under the Japanese long-term care insurance system: a cross-sectional analysis. PLoS One 2018;13:e0194919.

23 Robison J, Fortinsky R, Kleppinger A, et al. A broader view of family caregiving: effects of caregiving and caregiver conditions on depressive symptoms, health, work, and social isolation. $J$ Gerontol B Psychol Sci Soc Sci 2009;64:788-98.

24 Janus AL, Ermisch J. Who pays for home care? A study of nationally representative data on disabled older Americans. BMC Health Serv Res 2015;15:1-14.

25 Paraponaris A, Davin B, Verger P. Formal and informal care for disabled elderly living in the community: an appraisal of French care composition and costs. Eur J Health Econ 2012;13:327-36.

26 Kurth B-M, Lange C, Kamtsiuris P. Gesundheitsmonitoring am Robert Koch-Institut: Sachstand und Perspektiven. Bundesgesundheitsblatt - Gesundheitsforschung - Gesundheitsschutz 2009;52:557-70.

27 Kamtsiuris P, Lange M, Hoffmann R. Die erste Welle Der studie Zur Gesundheit Erwachsener in Deutschland (DEGS1): Stichprobendesign, response, Gewichtung und Repräsentativität. Bundesgesundheitsblatt - Gesundheitsforschung Gesundheitsschutz 2013;56:620-30.

28 Scheidt-Nave C, Kamtsiuris P, Gößwald A, et al. German health interview and examination survey for adults (DEGS) - design, objectives and implementation of the first data collection wave. BMC Public Health 2012;12:730-46.

29 Gößwald A, Lange M, Kamtsiuris P. Degs: studie Zur Gesundheit Erwachsener in Deutschland. Bundesweite Quer- und 
Längsschnittstudie Im Rahmen des Gesundheitsmonitorings des Robert Koch-Instituts. Bundesgesundheitsblatt -

Gesundheitsforschung - Gesundheitsschutz 2012;55:775-80.

30 Gößwald A, Lange M, Dölle R. Die erste Welle Der studie Zur Gesundheit Erwachsener in Deutschland (DEGS1): Gewinnung von Studienteilnehmenden, Durchführung Der Feldarbeit und Qualitätsmanagement. Bundesgesundheitsblatt Gesundheitsforschung - Gesundheitsschutz 2013;56:611-9.

31 Schlack R, Rüdel J, Karger A, et al. Körperliche und psychische Gewalterfahrungen in der deutschen Erwachsenenbevölkerung. Bundesgesundheitsblatt Gesundheitsforschung Gesundheitsschutz 2013:56:755-64.

32 Brauns H, Scherer S, Steinmann S. The CASMIN educational classification in international comparative research. In: HoffmeyerZlotnik JHP, Wolf C, eds. Advances in cross-national comparison. Boston, MA: Springer US, 2003: 221-44.

33 Lampert T, Kroll LE, der LEvon. Sozioökonomischer status und Gesundheit: Ergebnisse Der studie Zur Gesundheit Erwachsener in Deutschland (DEGS1). Bundesgesundheitsblatt Gesundheitsforschung - Gesundheitsschutz 2013:56:814-21.

34 Löwe B, Kroenke K, Gräfe K. Detecting and monitoring depression with a two-item questionnaire (PHQ-2). J Psychosom Res 2005;58:163-71.

35 Hapke U, Maske UE, Scheidt-Nave C. Chronischer stress bei Erwachsenen in Deutschland: Ergebnisse der studie zur Gesundheit Erwachsener in Deutschland (DEGS1). Bundesgesundheitsblatt - Gesundheitsforschung Gesundheitsschutz 2013;56:749-54.

36 Etters L, Goodall D, Harrison BE. Caregiver burden among dementia patient caregivers: a review of the literature. J Am Acad Nurse Pract 2008;20:423-8.

37 Räker M, Schwinger A, Klauber J. Was leisten ambulante Pflegehaushalte? Eine Befragung zu Eigenleistungen und finanziellen Aufwänden. In: Jacobs K, Kuhlmey A, Greß S, et al, eds. Pflege-Report 2020. Berlin, Heidelberg: Springer Berlin Heidelberg, 2020: 65-95.

38 Gottschalk S, König H-H, Brettschneider C. The association between informal caregiving and behavioral risk factors: a cross-sectional study. Int J Public Health 2020;65:911-21.

39 Hoffman GJ, Lee J, Mendez-Luck CA. Health behaviors among baby Boomer informal caregivers. Gerontologist 2012;52:219-30.

40 Roth DL, Brown SL, Rhodes JD, et al. Reduced mortality rates among caregivers: does family caregiving provide a stress-buffering effect? Psychol Aging 2018;33:619-29.

41 Beesley VL, Price MA, Webb PM, et al. Loss of lifestyle: health behaviour and weight changes after becoming a caregiver of a family member diagnosed with ovarian cancer. Support Care Cancer 2011;19:1949-56. 